\title{
Implication of lipid metabolism disturbance and Alzheimer's disease: focus on the lipoprotein lipase plays an important role in learning and memory function
}

\author{
Dehua Chui*, Ting Zhou, Liang Zhou, Huan Yang, Xinying Liu, Tingting Liu, Jia Yu, Yi Liu, Xue Fei Wu, Hui Zhang, \\ Dongsheng Fan
}

From 2011 International Conference on Molecular Neurodegeneration

Shanghai, China. 22-24 September 2011

\section{Background}

Along with aging, lipid metabolism disturbance occurs. Lipoprotein lipase (LPL) is also expressed in the brain with highest levels found in the pyramidal cells of the hippocampus, suggesting a possible role for LPL in the regulation of cognitive function. However, very little is currently known about the specific role of LPL in the brain.

\section{Method}

LPL deficient mice and littermate control C57BL/6J mice were bred in the animal facility of Peking University Health Science Center. The histochemistry and western blotting was performed as our previous description.

\section{Results}

In this study, we found that LPL-deficient mice exhibited increased latency to escape platform and increased mistake frequency. Decreased latency to platform in the stepdown passive avoidance test was observed, consistent with impaired learning and memory in these mice. Transmission electron microscopy revealed a significant decrease in the number of pre-synaptic vesicles in the hippocampus of LPL-deficient mice. The levels of the pre-synaptic marker synaptophysin were also reduced in the hippocampus while post-synaptic marker PSD-95 levels remained unchanged in LPL-deficient mice. LPL deficiency reduced the frequency of miniature excitatory postsynaptic currents (mEPSCs) and readily releasable pool (RRP) size. Then we demonstrated that these defects, which resulted from slower clathrin-mediated endocytosis and synaptic vesicle recycling, led to presynaptic dysfunction and synaptic plasticity impairment. Moreover, lipid assay revealed a lack of docosahexaenoic acid (DHA) and arachidonic acid (AA) in LPL deficient neurons. Meanwhile, exogenous DHA and AA partially rescued the defect of synaptic vesicle recycling in LPL deficient neurons.

\section{Conclusion}

This finding reveals a novel role of LPL in synaptic plasticity and contributes to a better understanding of the LPL function in the brain, where altered LPL levels are related to learning and memory impairment.

\section{Acknowledgment \\ This work was supported by the National Natural Science Foundation of China (NSFC; Grants No.30973145), Doctoral Fund of Ministry of Education (20090001110058) and the National High Technology Research and Development Programof China (973 Program, No. 2012CB911004).}

Published: 7 February 2012

doi:10.1186/1750-1326-7-S1-O9

Cite this article as: Chui et al:: Implication of lipid metabolism disturbance and Alzheimer's disease: focus on the lipoprotein lipase plays an important role in learning and memory function. Molecular Neurodegeneration 2012 7(Suppl 1):09.

\footnotetext{
* Correspondence: dchui@bjmu.edu.cn

Peking University Neuroscience Research Institute, Dep. of Neurology, Peking University Third Hospital, Beijing 100191, China
}

(c) 2012 Chui et al; licensee BioMed Central Ltd. This is an Open Access article distributed under the terms of the Creative Commons Attribution License (http://creativecommons.org/licenses/by/2.0), which permits unrestricted use, distribution, and reproduction in any medium, provided the original work is properly cited. 